\title{
Association of urinary RBP4 with insulin resistance, inflammation, and microalbuminuria
}

\section{Se Eun Park, Nam Seok Lee', Ji Woo Park' ${ }^{1}$ Eun-Jung Rhee, Won-Young Lee, Ki-Won Oh, Sung-Woo Park, Cheol-Young Park and Byung-Soo Youn ${ }^{2,3}$}

Division of Endocrinology and Metabolism, Department of Internal medicine, Kangbuk Samsung Hospital, Sungkyunkwan University School of Medicine, Seoul, Republic of Korea, ${ }^{1}$ AdipoGen, Inc., Room 401, Venture Building B, Songdo Technopark, 7-50 Songdo-dong, Yeonsu-gu, Incheon, Republic of Korea, ${ }^{2}$ Department of Anatomy, Wonkwang University School of Medicine, \#22 Wonkwang Daehak-ro, Iksan, Jeollabuk-do 570-749, Republic of Korea and ${ }^{3}$ OsteoNeuroGen 40 MiKeum-RO, Bundang-Gu, Sungnam-City, Gyeonggi-do 461-871, Republic of Korea
Correspondence should be addressed to C-Y Park or B-S Youn Email

cydoctor@chol.com or byung4jc@gmail.com

\begin{abstract}
Objective: Serum concentrations of retinol-binding protein 4 (RBP4) are elevated in type 2 diabetes and associated with the severity of insulin resistance; however, there are few data about the relationship between urinary RBP4 levels and metabolic parameters. We assessed urinary RBP4 as a new biomarker by establishing its relationship with clinical parameters associated with insulin resistance and urinary albumin excretion.

Design and methods: We measured RBP4 in the serum and urine of 689 subjects with diverse glucose tolerance status. We also evaluated the relationship between urinary RBP4 and cardiometabolic risk factors, including insulin resistance, high-sensitivity C-reactive protein (hsCRP), arterial stiffness, and microalbuminuria.

Results: Urinary RBP4 levels were higher in insulin-resistant subjects with prediabetes or type 2 diabetes than in subjects with normal glucose tolerance (NGT) (type 2 diabetes $>$ prediabetes $>$ NGT; all $P<0.001$ ). Urinary RBP4 correlated strongly with homeostasis model assessments of insulin resistance (HOMA-IR), fasting glucose, triglycerides, blood pressure, hsCRP, arterial stiffness, estimated glomerular filtration rate, and urinary albumin-to-creatinine ratio (all $P<0.01$ ). HOMA-IR and arterial stiffness were found to be independent determinants of urinary RBP4 concentration. Furthermore, urinary RBP4 was highly predictive of microalbuminuria (odds ratio $2.6,95 \% \mathrm{Cl} 1.6-4.2$ ), even after adjustment for other metabolic parameters. The area under the ROC curve for urinary RBP4 to detect the presence of microalbuminuria was $0.80 \pm 0.02$ (95\% Cl 0.76-0.84) and the cut-off value was $157.01 \mu \mathrm{g} / \mathrm{gCr}$.

Conclusions: Urinary RBP4 concentrations were elevated in patients with dysregulation of glucose metabolism and were related to various cardiometabolic risk factors including insulin resistance, inflammation, and microalbuminuria.
\end{abstract}

\section{Introduction}

Insulin resistance contributes to the progression from normal glucose tolerance (NGT) to impaired fasting glucose (IFG), impaired glucose tolerance (IGT), and type 2 diabetes mellitus (T2DM) (1). Even in the absence of chronic hyperglycemia or diabetes, insulin resistance confers an increased risk of cardiovascular disease (1). Serum retinol-binding protein (RBP4) is increased in insulin-resistant states and highly associated with the magnitude of insulin resistance and the severity of metabolic syndrome in humans $(2,3,4)$. Increased serum RBP4 causes insulin resistance in mice by interfering with insulin signaling in the
(C) 2014 European Society of Endocrinology Printed in Great Britain
Published by Bioscientifica Ltd. 
skeletal muscle and liver (2), and may exert similar actions in humans.

Microalbuminuria is an early marker of diabetic nephropathy and an independent risk factor for cardiovascular disease $(5,6)$. Previous data have demonstrated that microalbuminuria is associated with insulin resistance in both diabetic patients and nondiabetic individuals (7), and is therefore also an indicator of early-stage metabolic abnormalities involved in the pathogenesis of renal and cardiovascular disease. Serum RBP4 levels are also closely related to urinary albumin excretion $(8,9)$, although this relationship has not been fully elucidated. As RBP4 is primarily cleared by the kidney, serum RBP4 concentrations are affected by the glomerular filtration rate and become greatly elevated in end-stage renal disease in humans or after nephrectomy in rodents $(10,11,12,13)$. Nevertheless, the relationship between RBP4 excreted in urine and insulin sensitivity, glycemia, and other components of the metabolic syndrome has not been determined.

The main purpose of this study was to clarify the association between urinary RBP4 levels and various cardiometabolic risk factors, including insulin resistance, high-sensitivity C-reactive protein (hsCRP), and arterial stiffness. In addition, we evaluated whether circulating concentrations of RBP4 in the urine are related to the severity of microalbuminuria.

\section{Subjects and methods}

From September 2007 to September 2008, subjects aged 21-80 years with a history of hyperglycemia (fasting plasma glucose $\geq 5.5 \mathrm{mmol} / \mathrm{ml}$ ) or diabetes were enrolled in the Seoul Metro-City Diabetes Prevention Program (14). From five public health centers, we recruited a total of 689 subjects with NGT $(n=75)$, prediabetes (IFG and/or IGT, $n=143)$, or T2DM $(n=471)$; all subjects had stable renal function and serum creatinine $(\mathrm{Cr})$ concentrations less than $2.0 \mathrm{mg} / \mathrm{dl}$. The subjects with end-stage renal disease were excluded. The subjects were excluded if they were taking antiobesity medications or corticosteroids or if they had a history of diabetic ketoacidosis, symptomatic heart failure, or hepatic dysfunction. Assignment to groups was determined by a 75-g oral glucose tolerance test according to the diagnostic criteria of the American Diabetes Association (15). All participants provided written informed consent. This study was approved by the Institutional Review Board of Kangbuk Samsung Hospital.

Blood samples were collected after an overnight fast. The subjects provided a concurrent morning specimen of urine. Aliquots of plasma and urine were stored at $-80^{\circ} \mathrm{C}$.
Blood pressure, height, weight, and waist-and-hip circumferences were measured using standard methods. Plasma glucose concentration was determined using a Beckman glucose analyzer II (Beckman Instruments, Fullerton, CA, USA). HbA1c was measured using HPLC (Variant II, BioRad Laboratories). Intra-assay coefficient of variation (CV) values were $<1.5 \%$ for glucose and $<3.0 \%$ for HbA1c. Serum insulin was measured using an IRMA (Biosource, Nivells, Belgium) according to the manufacturer's recommendation. Plasma lipids, including total cholesterol, triglycerides (TG), HDL, and LDL, were measured by enzymatic colorimetric assay (ADVIA 1650). Serum hsCRP levels were measured by nephelometric assay using a BNII nephelometer (Dade Behring, Deerfield, IL, USA); hsCRP results were presented as milligrams per liter, and the limit of measurement was $0.175 \mathrm{mg} / \mathrm{l}$ with a sample dilution of 1:20. Homeostasis model assessments of insulin resistance (HOMA-IR) were calculated as described previously (16). Serum Cr concentrations were measured using the timed end-point method (Unicel DxC 800, Beckman and Coulter, Krefeld, Germany).

The urinary microalbumin-to-creatinine (albuminto-creatinine ratio, ACR) ratio was calculated using concurrent measurements in a morning daytime urine specimen by the immunonephelometric method using a DCA 2000 albumin:creatinine ratio urine analyzer (Bayer Corp.). Microalbuminuria was defined as an ACR of $30-300 \mu \mathrm{g} / \mathrm{mg}$ creatinine. Glomerular filtration rate was estimated by the Modification of Diet in Renal Disease Study Group formula (estimated glomerular filtration rate, eGFR) (17).

Urinary and serum RBP4 concentrations were measured using a commercial ELISA kit (Adipogen, Seoul, Korea). Intra-assay $\mathrm{CV}$ values for serum ranged between 2.02 and 3.59\%, and inter-assay CV values for serum ranged from 3.99 to $5.94 \%$. The intra-assay CV values for urine RBP4 ranged between 3.02 and $6.01 \%$, whereas the interassay $\mathrm{CV}$ values for urine RBP4 ranged between 5.87 and $10.16 \%$. This ELISA is capable of detecting both truncated and degraded RBP4 forms as well. The concentrations of ACR and RBP4 in urine were measured using the same samples. Serum transthyretin (TTR) levels were measured using an ELISA kit (ALPCO Diagnostics, Windham, NH, USA).

Pulse wave velocity (PWV) was determined using an automatic waveform analyzer VP2000 (Omron Healthcare Co., Ltd., Kyoto, Japan). The subjects were examined in the supine position after resting for at least $5 \mathrm{~min}$. The pressure waveforms of the brachial and tibial arteries were recorded by an oscillometric method using 
occlusion/sensing cuffs applied to both arms and both ankles. We used the mean baPWV (average of right and left brachial-ankle PWVs) value as a marker of arterial stiffness.

\section{Statistical analyses}

Continuous variables with normal distribution were expressed as means \pm s.D., and variables with skewed distributions were expressed as median and range and log-transformed for statistical analysis. ANOVA, Wilcoxon rank-sum, and Kruskal-Wallis tests were used for comparison of continuous variables, and the $\chi^{2}$-test was used for comparison of categorical variables. Pearson's correlation analysis identified significant correlations between urinary RBP4 concentrations and various metabolic parameters. Stepwise linear regression analyses were used to identify independent predictors of RBP4 concentrations in urine. Multivariate logistic regression analyses were performed to test the independent strengths of associations of microalbuminuria or combined microalbuminuria and macroalbuminuria with urinary RBP4. ROC curves were plotted for the detection of microalbuminuria. Statistical analyses were conducted with SPSS 18.0 software for Windows (SPSS, Inc.). A $P$ value $<0.05$ was considered significant for all studies.

\section{Results}

\section{Urinary RBP4 concentrations in insulin-resistant human subjects and normal control subjects}

Anthropometric and clinical characteristics of study participants are shown in Table 1. With increasing categories of impaired glucose metabolism, we found the expected group differences in several metabolic parameters, including age, BMI, waist circumference, fasting blood glucose, HbA1c, TG, HDL, fasting insulin, insulin sensitivity (HOMA-IR), hsCRP, and blood pressure.

Table 1 Baseline characteristics of the study population. Data are summarized as mean \pm s.D., median (interquartile range) for non-normal distribution or $n(\%)$.

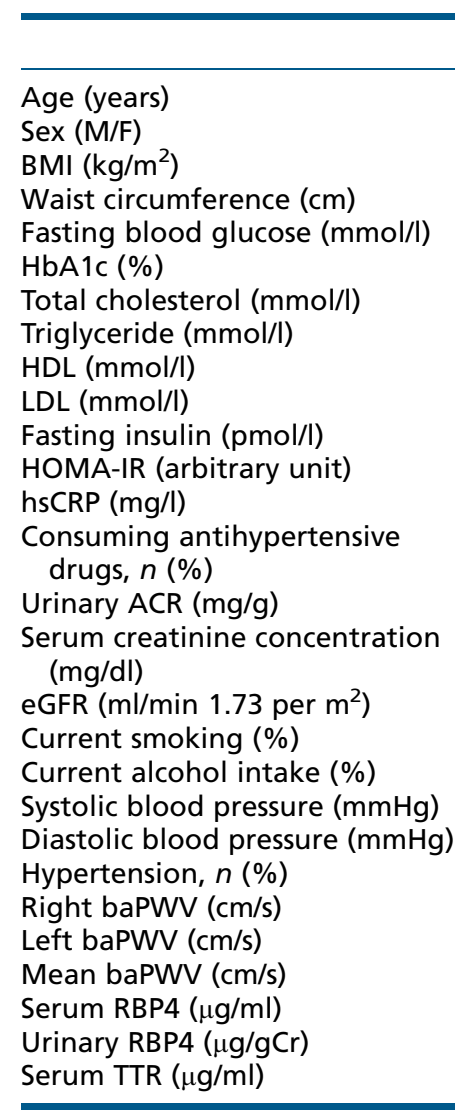

\begin{tabular}{c}
\hline Normal $(n=75)$ \\
\hline $40.28 \pm 0.977$ \\
$34 / 41$ \\
$22.63 \pm 3.21$ \\
$78.01 \pm 9.79$ \\
$5.17 \pm 0.28$ \\
$5.32 \pm 0.29$ \\
$4.62 \pm 0.78$ \\
$1.06 \pm 0.76$ \\
$1.40 \pm 0.32$ \\
$2.82 \pm 0.80$ \\
$38.76 \pm 19.43$ \\
$1.39 \pm 0.72$ \\
$0.13 \pm 0.28$ \\
$1(1.3)$ \\
$9.68 \pm 11.74$ \\
$0.85 \pm 0.19$
\end{tabular}

$94.77 \pm 20.54$

17.3

38.7

$113.19 \pm 9.86$

$70.05 \pm 7.59$

$1(1.3)$

$1199.6 \pm 203.6$

$1245.1 \pm 193.9$

$1822.1 \pm 298.3$

$73.86 \pm 32.81$

$100.91 \pm 64.65$

$173.86 \pm 88.46$

\begin{tabular}{c}
\hline IFG or IGT $(n=143)$ \\
\hline $57.83 \pm 12.78^{*}$ \\
$61 / 82$ \\
$24.55 \pm 3.62^{*}$ \\
$84.32 \pm 10.34^{*}$ \\
$6.16 \pm 0.39 *$ \\
$5.72 \pm 0.30$ \\
$5.09 \pm 0.93^{*}$ \\
$1.50 \pm 0.74 *$ \\
$1.37 \pm 0.30$ \\
$2.91 \pm .0 .83$ \\
$66.00 \pm 24.83^{*}$ \\
$2.80 \pm 1.12^{*}$ \\
$0.18 \pm .57$ \\
$50(35.0) *$
\end{tabular}

$28.94 \pm 54.50$ *

$0.73 \pm 0.19 *$

$94.26 \pm 30.15$

20.9

26.9

$123.45 \pm 15.89$ *

$73.27 \pm 10.01$ *

$50(35.0) *$

$1481.0 \pm 347.2$ *

$1483.3 \pm 435.7$ *

$2222.7 \pm 528.5^{*}$

$108.17 \pm 59.02$

$391.08 \pm 723.33$ *

$495.52 \pm 768.14$ *

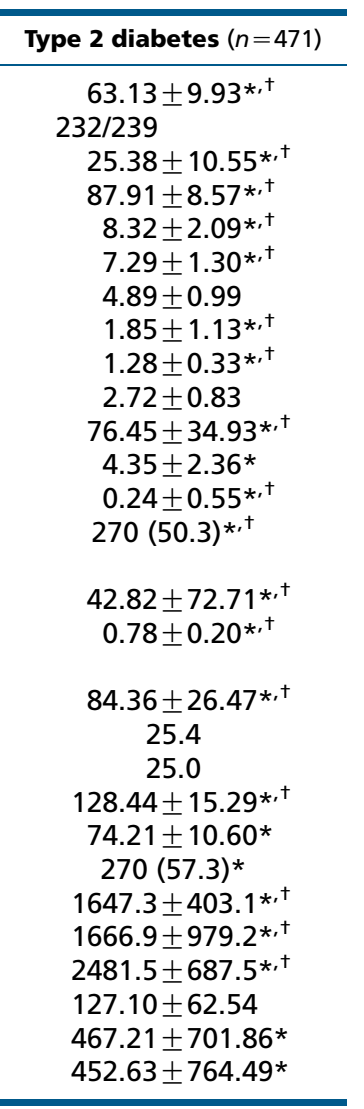

$\begin{array}{r}r \text { P value } \\ \hline<0.001 \\ 0.355 \\ 0.041 \\ <0.001 \\ <0.001 \\ <0.001 \\ 0.003 \\ <0.001 \\ <0.001 \\ 0.051 \\ <0.001 \\ <0.001 \\ <0.001 \\ <0.001 \\ \\ <0.001 \\ <0.001 \\ \\ <0.001 \\ 0.220 \\ 0.047 \\ <0.001 \\ <0.001 \\ <0.001 \\ <0.001 \\ <0.001 \\ <0.001 \\ <0.001 \\ <0.001 \\ 0.013 \\ \hline\end{array}$

${ }^{*} P<0.005$ vs normal group; ${ }^{\dagger} P<0.005$ vs IFG/IGT group; IFG, impaired fasting glucose; IGT, impaired glucose tolerance; HOMA-IR, homeostatic model assessment-insulin resistance; hsCRP, high-sensitivity C-reactive protein; ACR, albumin-to-creatinine ratio; eGFR, estimated glomerular filtration rate; PWV, pulse wave velocity; RBP, retinol-binding protein; TTR, transthyretin. 
Urinary ACR also increased with worsening insulinglucose homeostasis (i.e. from NGT to prediabetes and from prediabetes to T2DM), but only the subjects with T2DM exhibited impaired renal function as a group, as indicated by significantly increased serum $\mathrm{Cr}$ and reduced eGFR. Both side and mean baPWV progressively increased from NGT to prediabetes to T2DM in a stepwise manner (Table 1). Urinary RBP4 levels were greater in subjects with prediabetes and T2DM than in subjects with NGT (T2DM $>$ prediabetes $>$ NGT, $P<0.001$ for all; Table 1 ). Serum TTR concentrations followed a similar trend $(P=0.013$ for all; Table 1$)$.

\section{Correlation between urinary RBP4 concentrations and metabolic parameters}

Univariate regression analyses revealed significant relationships between urinary RBP4 concentrations and metabolic parameters. Urinary levels of RBP4 were associated with serum RBP4 and TTR levels, consistent with its proposed function in stabilization of RBP4 in the serum (18). Both urinary and serum RBP4 levels correlated positively with age, waist circumference, fasting glucose, TG, fasting insulin, HOMA-IR, eGFR, and urinary ACR in all subjects. Serum RBP4 levels were also positively associated with total cholesterol levels. Although PWV correlated with both urinary RBP4 and serum RBP4 levels, blood pressure and hsCRP levels correlated only with urinary RBP4, and not with serum RBP4 (Table 2). Stepwise linear regression analysis revealed that HOMA-IR and mean PWV were strong predictors for urinary RBP4 levels, but age, sex, BMI, TG, systolic blood pressure, hsCRP, and eGFR were excluded (Table 3).

\section{Association between urinary RBP4 concentrations and albuminuria}

Multivariate regression analysis models indicated that urinary RBP4 concentrations (log(urinary RBP4( $\mu \mathrm{g} / \mathrm{gCr}))$ ) strongly predicted an increased risk for microalbuminuria (odds ratio (OR) 13.22, 95\% CI 7.78-22.47, $P<0.001$ ) and combined micro- and macroalbuminuria (OR 12.30, 95\% CI 7.42-20.32, $P<0.001$ ), even after adjusting for age, sex, BMI, current status of smoking and alcohol intake, blood pressure, use of angiotensin receptor blocker or angiotensin-converting enzyme inhibitor, HOMA-IR, triglyceride, hsCRP, and eGFR. Urinary RBP4 concentration showed a stronger association with urinary ACR than serum RBP4

Table 2 Correlation between serum or urinary RBP4 and various metabolic parameters.

\begin{tabular}{|c|c|c|c|c|}
\hline & \multicolumn{2}{|c|}{ Urinary RBP4 $(\mu \mathrm{g} / \mathrm{gCr})^{\mathrm{a}}$} & \multicolumn{2}{|c|}{ Serum RBP4 $(\mu \mathrm{g} / \mathrm{ml})$} \\
\hline & Correlation coefficient & $P$ & Correlation coefficient & $P$ \\
\hline Age (years) & 0.272 & $<0.001$ & 0.139 & $<0.001$ \\
\hline BMI $\left(\mathrm{kg} / \mathrm{m}^{2}\right)$ & -0.005 & 0.889 & 0.022 & 0.562 \\
\hline Waist circumference $(\mathrm{cm})$ & 0.132 & 0.001 & 0.139 & $<0.001$ \\
\hline Fasting glucose (mmol/l) & 0.286 & $<0.001$ & 0.241 & $<0.001$ \\
\hline Total cholesterol (mmol/l) & 0.042 & 0.271 & 0.092 & 0.016 \\
\hline Triglyceride $(\mathrm{mmol} / \mathrm{l})^{\mathrm{a}}$ & 0.113 & $<0.003$ & 0.351 & $<0.001$ \\
\hline $\mathrm{HDL}(\mathrm{mmol} / \mathrm{l})$ & -0.011 & 0.778 & -0.056 & 0.140 \\
\hline $\mathrm{LDL}(\mathrm{mmol} / \mathrm{l})$ & 0.001 & 0.980 & -0.020 & 0.598 \\
\hline Fasting insulin $(\mathrm{pmol} / \mathrm{l})^{\mathrm{a}}$ & 0.159 & $<0.001$ & 0.163 & $<0.001$ \\
\hline HOMA-IR (arbitrary unit) ${ }^{a}$ & 0.242 & $<0.001$ & 0.239 & $<0.001$ \\
\hline $\mathrm{HsCRP}(\mathrm{mg} / \mathrm{l})^{\mathrm{a}}$ & 0.114 & $<0.001$ & -0.023 & 0.558 \\
\hline Urinary ACR $(\mathrm{mg} / \mathrm{g})^{\mathrm{a}}$ & 0.576 & $<0.001$ & 0.134 & 0.001 \\
\hline $\begin{array}{l}\text { Serum creatinine concentration } \\
(\mathrm{mg} / \mathrm{dl})\end{array}$ & 0.020 & 0.592 & 0.288 & $<0.001$ \\
\hline Systolic blood pressure (mmHg) & 0.179 & $<0.001$ & 0.038 & 0.348 \\
\hline Diastolic blood pressure $(\mathrm{mmHg})$ & 0.124 & $<0.001$ & 0.062 & 0.127 \\
\hline eGFR $\left(\mathrm{ml} / \mathrm{min}\right.$ per $\left.1.73 \mathrm{~m}^{2}\right)$ & -0.113 & 0.003 & -0.203 & $<0.001$ \\
\hline Right baPWV $(\mathrm{cm} / \mathrm{s})^{a}$ & 0.195 & $<0.001$ & 0.116 & $<0.001$ \\
\hline Left baPWV $(\mathrm{cm} / \mathrm{s})^{a}$ & 0.184 & $<0.001$ & 0.099 & $<0.001$ \\
\hline Mean baPWV $(\mathrm{cm} / \mathrm{s})^{a}$ & 0.194 & $<0.001$ & 0.103 & 0.011 \\
\hline Serum RBP4 $(\mu \mathrm{g} / \mathrm{ml})$ & 0.173 & $<0.001$ & - & - \\
\hline Urinary RBP4 $(\mu \mathrm{g} / \mathrm{gCr})$ & - & - & 0.132 & 0.001 \\
\hline Serum TTR $(\mu \mathrm{g} / \mathrm{ml})^{\mathrm{a}}$ & 0.091 & 0.017 & 0.167 & $<0.001$ \\
\hline
\end{tabular}

HOMA-IR, homeostatic model assessment insulin resistance; hsCRP, high-sensitivity C-reactive protein; ACR, albumin-to-creatinine ratio; eGFR, estimated glomerular filtration rate; PWV, pulse wave velocity; RBP, retinol-binding protein; TTR, transthyretin.

${ }^{a}$ Logarithmic transformation was performed because of skewed distribution. 
Table 3 Stepwise linear regression of independent predictors of log(urinary RBP4 $(\mu \mathrm{g} / \mathrm{gCr})$ ) concentration.

\begin{tabular}{|c|c|c|c|}
\hline \multirow[b]{2}{*}{ Variables } & \multicolumn{3}{|c|}{ Urinary RBP4 $(\mu \mathrm{g} / \mathrm{gCr})^{\mathrm{a}}$} \\
\hline & Coefficient & S.E.M. & $P$ \\
\hline $\begin{array}{l}\text { HOMA-IR (arbitrary unit) } \\
\text { Mean PWV (cm/s) }\end{array}$ & $\begin{array}{l}0.216 \\
0.948\end{array}$ & $\begin{array}{l}0.092 \\
0.201\end{array}$ & $\begin{array}{r}0.020 \\
<0.001\end{array}$ \\
\hline
\end{tabular}

Independent variables included were age, sex, BMI, HOMA-IR ${ }^{a}$, triglyceride $^{a}$, systolic blood pressure, hsCRPa ${ }^{a}$ eGFR, and mean PWV ${ }^{\text {a }}$. The dependent variable was urinary RBP4 $(\mu \mathrm{g} / \mathrm{gCr})$. HOMA-IR, homeostatic model assessment insulin resistance; eGFR, estimated glomerular filtration rate; PWV, pulse wave velocity; RBP, retinol-binding protein.

${ }^{a}$ This variable was log-transformed before analysis.

concentration (OR 1.004, 95\% CI 1.000-1.007, $P=0.032$ for microalbuminuria and OR 1.004, 95\% CI 1.000-1.007, $P=0.032$ for combined micro- and macroalbuminuria). The area under the ROC curve for urinary RBP4 to detect the presence of microalbuminuria was $0.80 \pm 0.02$ (95\% CI 0.76-0.84, Fig. 1). The cutoff value of urinary RBP4 was $157.01 \mu \mathrm{g} / \mathrm{gCr}$ and the sensitivity was $80.18 \%$, and the specificity was $64.03 \%$.

\section{Discussion}

In this study, we show that urinary RBP4 concentrations were elevated in patients with dysregulation of glucose metabolism and were associated with several cardiometabolic parameters including insulin resistance, inflammation, and arterial stiffness. In addition, urinary RBP4 was strongly predictive of urinary albumin excretion, even in early stages of renal disease before the development of macroalbuminuria and reduced eGFR levels characteristic of advanced diabetic nephropathy. Urinary RBP4 may therefore be a particularly useful screening tool for metabolic disease in populations at risk. Urine studies are noninvasive, and this assay can be carried out in parallel with other urine studies such as ACR that utilize random 'spot' urine samples.

RBP4 is upregulated in the adipose tissue of several insulin resistant mouse models and causes hyperglycemia by interfering with insulin signaling in skeletal muscle and the liver $(2,3)$. Elevated serum RBP4 levels are associated with the severity of insulin resistance, obesity, and components of the metabolic syndrome in humans (4). This study extends previous findings by showing that both serum and urinary RBP4 levels are positively correlated with a number of metabolic parameters related with insulin resistance as well as an insulin resistance index. The insulin resistance index was also found to be an independent determinant of urinary RBP4 concentration.
Consistent with previous reports $(8,19)$, our data indicate that elevated serum RBP4 concentrations might lead to increased urinary excretion of RBP4. Further studies are required to determine whether urinary RBP4 excretion plays a causal role in the regulation of serum RBP4 levels and insulin resistance.

Low-grade systemic inflammation, possibly resulting from macrophage infiltration in adipose tissue, has been shown to correlate with both increased RBP4 expression in adipose tissue and increased circulating RBP4 (20). The associations between RBP4 and markers of systemic inflammation (20) have been also described in obese individuals, and inflammation represents a potential mediator of RBP4associated metabolic alterations (21). HsCRP is a recognised marker of low-grade inflammation in obesity and other insulin-resistant states. We found that urinary RBP4 excretion positively correlated with serum hsCRP levels, but serum RBP4 concentrations did not. To our knowledge, this is the first study to demonstrate a relationship between urinary RBP4 excretion and the systemic inflammation associated with insulin resistance in human subjects.

We found that urinary albumin excretion was significantly associated with urinary RBP4 concentrations in patients with relatively intact renal function. Urinary excretion of RBP4 has been recommended as a useful marker for the detection of minor changes in proximal tubular function before the occurrence of other markers such as overt proteinuria or an increase in plasma creatinine (22). An increase in the filtered load of albumin may be

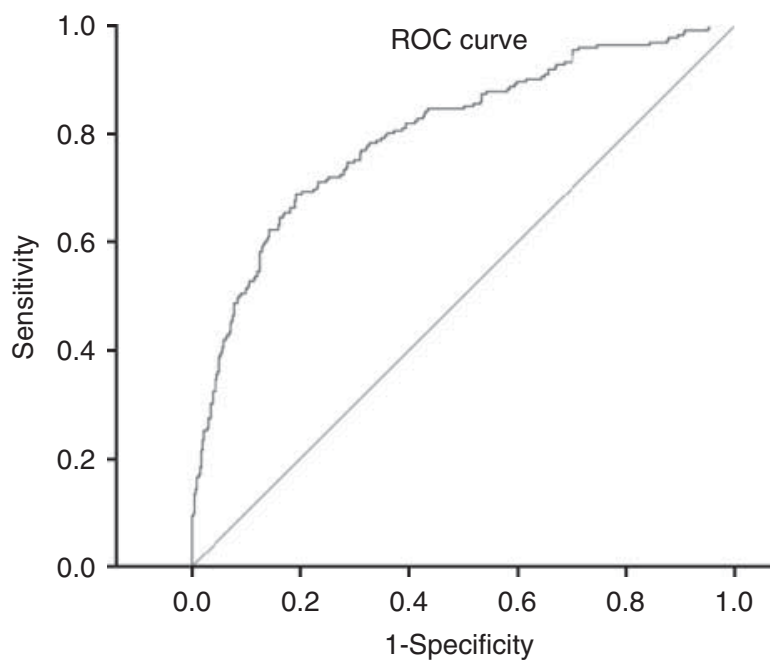

Figure 1

ROC analysis of urinary RBP4 levels for the prediction of microalbuminuria. 
responsible for the increased urinary excretion of RBP4 as a result of competition for reabsorption sites in the proximal tubules (23), leading to the positive correlation between urinary RBP4 and urinary albumin levels observed in our study. Previously, it was reported that serum RBP4 levels are also an independent risk factor for microalbuminuria $(8,9)$. Urinary RBP4 levels appeared to be more effective than serum RBP4 levels for detecting microalbuminuria. We showed that urinary RBP4 level might be an effective biomarker for insulin resistance and inflammation as well as microalbuminuria, although these results could not suggest that urinary RBP4 is not a more sensitive biomarker than urinary albumin. Further study is needed to compare the urinary RBP4 with urinary albumin as a biomarker of diabetic nephropathy.

Macrophage accumulation in diabetic kidneys predicts declining renal function and inflammatory cells might have a pathogenic role in diabetic nephropathy (24). Inflammation is also known to be associated with an increased risk for cardiovascular disease (25), and it was reported that higher serum RBP4 levels are associated with an increased risk of coronary heart disease (26). Insulin resistance is also closely associated with an increased risk of cardiovascular disease (1). In our study, arterial stiffness, measured using PWV, a useful marker for the assessment of increased cardiovascular risk (27), was found to be an independent determinant of urinary RBP4 concentration. Furthermore, we found that blood pressure, a well-known risk factor for cardiovascular disease, was associated with urinary RBP4 levels, not with serum RBP4 levels. In the current study, the significant association between urinary RBP4 and cardiovascular risk factors might be closely related to the link of urinary RBP4 with insulin resistance, inflammation, and microalbuminuria. Previously, the relationships between arterial stiffness and albuminuria were reported in type 2 diabetic patients (28). Generalized vascular damage may serve as a common pathogenic mechanism linking microalbuminuria and premature atherosclerosis (29). These findings suggest that urinary RBP4 as well as microalbuminuria might reflect generalized vascular dysfunction and be a useful marker for cardiovascular risk factors.

Because of the cross-sectional nature of our study, we were not able to examine the temporal relation between urinary RBP4, insulin resistance, and related metabolic parameters. A long-term follow up of larger groups will be needed to establish a predictive relationship between urinary RBP4 concentrations and metabolic parameters and renal function. In addition, our study subjects were not drug-naïve, and some subjects were on antihypertensive and/or glucose-lowering medications, which could influence both RBP4 concentrations and renal excretion of
RBP4 and/or albumin. However, the stochastic effects of different drug combinations in our patients would most likely result in weaker correlations between urinary RBP4 and metabolic and renal function parameters. Therefore, it is possible that these relationships may be even stronger in drug-naïve subjects.

Despite these limitations, our results provide important evidence that urinary RBP4 concentrations are elevated in insulin-resistant patients with prediabetes and T2DM, and that urinary RBP4 excretion correlates with the magnitude of insulin resistance, inflammation, and urinary albumin excretion. As RBP4 excretion is known to be increased in the setting of renal proximal tubular injury $(8,23)$, it is possible that urinary RBP4 excretion will provide additional information for stratifying the risk of renal disease progression among subjects with insulin resistance and type 2 diabetes. Prospective studies will be needed to further define the potential role of urinary RBP4 measurement in the diagnosis and management of insulin-resistant patients.

\section{Declaration of interest}

N S Lee and J W Park are employed by AdipoGen, Inc. B-S Youn is employed by OsteoNeuroGen. Other authors have no personal or financial conflicts of interest.

\section{Funding}

This work was supported in part by a grant from Ministry for Health, Welfare and Family Affairs (A081023 to B-S Youn and C-Y Park), Republic of Korea.

\section{Acknowledgements}

The authors thank Barbara B Kahn, Timothy E Graham, and Matthias Blüher for initial collaboration on the manuscript.

\section{References}

1 Reaven GM. Insulin resistance, the insulin resistance syndrome, and cardiovascular disease. Panminerva Medica 200547 201-210.

2 Yang Q, Graham TE, Mody N, Preitner F, Peroni OD, Zabolotny JM, Kotani K, Quadro L \& Kahn BB. Serum retinol binding protein 4 contributes to insulin resistance in obesity and type 2 diabetes. Nature 2005436 356-362. (doi:10.1038/nature03711)

3 Muoio DM \& Newgard CB. Metabolism: a is for adipokine. Nature 2005 436 337-338. (doi:10.1038/436337a)

4 Graham TE, Yang Q, Bluher M, Hammarstedt A, Ciaraldi TP, Henry RR, Wason CJ, Oberbach A, Jansson PA, Smith U et al. Retinol-binding protein 4 and insulin resistance in lean, obese, and diabetic subjects. New England Journal of Medicine 2006354 2552-2563. (doi:10.1056/ NEJMoa054862)

5 Stehouwer CD, Gall MA, Twisk JW, Knudsen E, Emeis JJ \& Parving HH. Increased urinary albumin excretion, endothelial dysfunction, and 
chronic low-grade inflammation in type 2 diabetes: progressive, interrelated, and independently associated with risk of death. Diabetes 200251 1157-1165. (doi:10.2337/diabetes.51.4.1157)

6 Mykkanen L, Zaccaro DJ, O'Leary DH, Howard G, Robbins DC \& Haffner SM. Microalbuminuria and carotid artery intima-media thickness in nondiabetic and NIDDM subjects. The Insulin Resistance Atherosclerosis Study (IRAS). Stroke 199728 1710-1716. (doi:10.1161/ 01.STR.28.9.1710)

7 Parvanova AI, Trevisan R, Iliev IP, Dimitrov BD, Vedovato M, Tiengo A, Remuzzi G \& Ruggenenti P. Insulin resistance and microalbuminuria: a cross-sectional, case-control study of 158 patients with type 2 diabetes and different degrees of urinary albumin excretion. Diabetes 200655 1456-1462. (doi:10.2337/db05-1484)

8 Raila J, Henze A, Spranger J, Mohlig M, Pfeiffer AF \& Schweigert FJ. Microalbuminuria is a major determinant of elevated plasma retinolbinding protein 4 in type 2 diabetic patients. Kidney International 2007 72 505-511. (doi:10.1038/sj.ki.5002372)

9 Xu M, Li XY, Wang JG, Wang XJ, Huang Y, Cheng Q, Huang HE, Li R, Xiang J, Tan JR et al. Retinol-binding protein 4 is associated with impaired glucose regulation and microalbuminuria in a Chinese population. Diabetologia 200952 1511-1519. (doi:10.1007/s00125-009-1386-8)

10 Graham TE, Wason CJ, Bluher M \& Kahn BB. Shortcomings in methodology complicate measurements of serum retinol binding protein (RBP4) in insulin-resistant human subjects. Diabetologia 2007 50 814-823. (doi:10.1007/s00125-006-0557-0)

11 Cabre A, Lazaro I, Girona J, Manzanares J, Marimon F, Plana N, Heras M \& Masana L. Retinol-binding protein 4 as a plasma biomarker of renal dysfunction and cardiovascular disease in type 2 diabetes. Journal of Internal Medicine 2007262 496-503. (doi:10.1111/j.1365-2796.2007. 01849.x)

12 Axelsson J, O'Byrne SM, Blaner WS, Carrero JJ, Bruchfeld A, Heimburger O, Barany P, Lindholm B \& Stenvinkel P. Serum retinolbinding protein concentration and its association with components of the uremic metabolic syndrome in nondiabetic patients with chronic kidney disease stage 5. American Journal of Nephrology 200929 447-453. (doi:10.1159/000176534)

13 Henze A, Frey SK, Raila J, Tepel M, Scholze A, Pfeiffer AF, Weickert MO, Spranger J \& Schweigert FJ. Evidence that kidney function but not type 2 diabetes determines retinol-binding protein 4 serum levels. Diabetes 200857 3323-3326. (doi:10.2337/db08-0866)

14 Won JC, Park CY, Park HS, Kim JH, Choi ES, Rhee EJ, Lee WY, Oh KW, Kim SW \& Park SW. 1,5-Anhydroglucitol reflects postprandial hyperglycemia and a decreased insulinogenic index, even in subjects with prediabetes and well-controlled type 2 diabetes. Diabetes Research and Clinical Practice 200984 51-57. (doi:10.1016/j.diabres. 2009.01.002)

15 Diagnosis and classification of diabetes mellitus. Diabetes Care 200528 S37-S42. (doi:10.2337/diacare.28.suppl_1.S37)
16 Matthews DR, Hosker JP, Rudenski AS, Naylor BA, Treacher DF \& Turner RC. Homeostasis model assessment: insulin resistance and $\beta$-cell function from fasting plasma glucose and insulin concentrations in man. Diabetologia 198528 412-419. (doi:10.1007/BF00280883)

17 Kuan Y, Hossain M, Surman J, El Nahas AM \& Haylor J. GFR prediction using the MDRD and Cockcroft and Gault equations in patients with end-stage renal disease. Nephrology, Dialysis, Transplantation 200520 2394-2401. (doi:10.1093/ndt/gfi076)

18 Zanotti G \& Berni R. Plasma retinol-binding protein: structure and interactions with retinol, retinoids, and transthyretin. Vitamins and Hormones 200469 271-295. (doi:10.1016/S0083-6729(04)69010-8)

19 Abahusain MA, Wright J, Dickerson JW \& de Vol EB. Retinol, $\alpha$-tocopherol and carotenoids in diabetes. European Journal of Clinical Nutrition 199953 630-635. (doi:10.1038/sj.ejcn.1600825)

20 Yao-Borengasser A, Varma V, Bodles AM, Rasouli N, Phanavanh B, Lee MJ, Starks T, Kern LM, Spencer HJ III, Rashidi AA et al. Retinol binding protein 4 expression in humans: relationship to insulin resistance, inflammation, and response to pioglitazone. Journal of Clinical Endocrinology and Metabolism 200792 2590-2597. (doi:10.1210/ jc.2006-0816)

21 Perseghin G, Petersen K \& Shulman GI. Cellular mechanism of insulin resistance: potential links with inflammation. International Journal of Obesity and Related Metabolic Disorders 200327 S6-11. (doi:10.1038/ sj.ijo.0802491)

22 Hong CY \& Chia KS. Markers of diabetic nephropathy. Journal of Diabetes and its Complications 199812 43-60. (doi:10.1016/S10568727(97)00045-7)

23 Birn H \& Christensen EI. Renal albumin absorption in physiology and pathology. Kidney International 200669 440-449. (doi:10.1038/ sj.ki.5000141)

24 Tesch GH. Macrophages and diabetic nephropathy. Seminars in Nephrology 201030 290-301. (doi:10.1016/j.semnephrol.2010.03.007)

25 Libby P. Inflammation and cardiovascular disease mechanisms. American Journal of Clinical Nutrition 200683 456S-460S.

26 Sun Q, Kiernan UA, Shi L, Phillips DA, Kahn BB, Hu FB, Manson JE, Albert CM \& Rexrode KM. Plasma retinol-binding protein 4 (RBP4) levels and risk of coronary heart disease: a prospective analysis among women in the nurses' health study. Circulation 2013127 1938-1947. (doi:10.1161/CIRCULATIONAHA.113.002073)

27 Zieman SJ, Melenovsky V \& Kass DA. Mechanisms, pathophysiology, and therapy of arterial stiffness. Arteriosclerosis Thrombosis and Vascular Biology 200525 932-943.

28 Smith A, Karalliedde J, De Angelis L, Goldsmith D \& Viberti G. Aortic pulse wave velocity and albuminuria in patients with type 2 diabetes. Journal of the American Society of Nephrology 200516 1069-1075.

29 Stehouwer CD, Nauta JJ, Zeldenrust GC, Hackeng WH, Donker AJ \& den Ottolander GJ. Urinary albumin excretion, cardiovascular disease, and endothelial dysfunction in non-insulin-dependent diabetes mellitus. Lancet 1992340 319-323.

Received 28 March 2014

Revised version received 30 June 2014

Accepted 3 July 2014 